\title{
Postsynaptic Signaling via the $\mu$-Opioid Receptor: Responses of Dorsal Horn Neurons to Exogenous Opioids and Noxious Stimulation
}

\author{
Jodie A. Trafton, Catherine Abbadie, Kurt Marek, and Allan I. Basbaum \\ Departments of Anatomy and Physiology and W. M. Keck Foundation for Integrative Neuroscience, University of California \\ San Francisco, San Francisco, California 94143
}

\begin{abstract}
Although both pre- and postsynaptic mechanisms have been implicated in the analgesia produced by $\mu$-opioids at the spinal cord, it is not known under what conditions these different controls come into play. Because the $\mu$-opioid receptor (MOR) can be visualized in individual lamina II excitatory interneurons and internalizes into endosomes on ligand binding, we tested whether MOR internalization could be monitored and used to measure postsynaptic MOR signaling. To test whether endogenous opioids modulate these lamina II interneurons during noxious stimulation, we next assessed the magnitude of postsynaptic MOR internalization under a variety of nociceptive conditions.

As observed in other systems, we show that MOR internalization in dorsal horn interneurons is demonstrated readily in response to opioid ligands. The MOR internalization is dosedependent, with a similar dose-response to that observed for opioid-induced increases in potassium conductance. We dem-
\end{abstract}

onstrate that MOR internalization in lamina II neurons correlates precisely with the extent of analgesia produced by intrathecal DAMGO. These results suggest that MOR internalization provides a good marker of MOR signaling in the spinal cord and that postsynaptic MORs on lamina II interneurons likely participate in the analgesia that is produced by exogenous opioids. We found, however, that noxious stimuli, under normal or inflammatory conditions, did not induce MOR internalization. Thus, endogenous enkephalins and endomorphins, thought to be released during noxious peripheral stimuli, do not modulate nociceptive messages via postsynaptic MORs on lamina II interneurons. We suggest that any endogenous opioids that are released by noxious stimuli target presynaptic MORs or $\delta$-opioid receptors.

Key words: receptor internalization; spinal cord; nociception; analgesia; inflammation; substantia gelatinosa
Although the ability of spinally administered $\mu$-opioids to produce analgesia is well documented, the mechanisms via which these opioids act are not understood fully. The $\mu$-opioid receptor (MOR) is expressed by primary afferent nociceptors that terminate in lamina I and II of the dorsal horn and by glutamatergic interneurons in lamina II (Arvidsson et al., 1995a). Based on this distribution and on functional studies of opioid-induced activity, two major mechanisms for producing opioid analgesia have been proposed: presynaptic inhibition of neurotransmitter release from primary afferent nociceptors and postsynaptic hyperpolarization of excitatory interneurons. Unclear, however, are the conditions when these two mechanisms come into play.

Like other G-protein-coupled receptors, the MOR internalizes into endosomes in vitro and in vivo on exposure to receptor agonists (Arden et al., 1995; Sternini et al., 1996). Because receptor internalization is agonist-dependent, it has been suggested that internalization of seven-transmembrane-domain receptors can be used as a marker of their activity (Mantyh et al., 1995). For example, MOR internalization has been used to document opioid activity induced by estrogen priming and exogenous opioids in hypothalamus and enteric neurons, respectively (Sternini et al., 1996; Eckersell et al., 1998). Unfortunately, because of the small size and

\footnotetext{
Received June 20, 2000; revised Aug. 28, 2000; accepted Sept. 11, 2000.

This research was supported by National Institute of Health Grants DA 08377, NS 21445, and NS 14627. J.T. was supported in part by a National Science Foundation predoctoral fellowship, an Achievement Award for College Scientist scholarship, and the Veterans Affairs Health Services Research and Development Service. C.A. was supported by Institut National de la Santé et de la Recherche Médicale, France and Institut UPSA de la douleur.

Correspondence should be addressed to Dr. Allan I. Basbaum, Departments of Anatomy and Physiology, University of California San Francisco, 513 Parnassus, Box 0452, San Francisco, CA 94143-0452. E-mail: aib@phy.ucsf.edu.

Dr. Trafton's present address: VA Health Services Research and Development Center for Health Care Evaluation, 795 Willow Road (152 MPD), Menlo Park, CA 94025 .

Dr. Abbadie's present address: Laboratory of Molecular Neuropharmacology, Memorial Sloan-Kettering Cancer Center, New York, NY 10021.

Copyright (C) 2000 Society for Neuroscience $0270-6474 / 00 / 208578-07 \$ 15.00 / 0$
}

punctate appearance of synaptic terminals, light microscopic examination of MOR internalization cannot be used to monitor presynaptic MOR activity. By contrast, it should be possible to observe the internalization of the postsynaptic MOR in interneurons in lamina II where membrane and intracellular labeling are easily distinguishable. Although the relationship between opioid receptor internalization and function is not well established, we hypothesized that if MOR internalization correlates with MOR-mediated postsynaptic inhibitory effects, then MOR internalization could be used to monitor MOR signaling by these interneurons in vivo.

There is evidence that spinal cord MORs can be activated endogenously by pain-producing stimuli. For example, neurochemical studies demonstrated that endogenous enkephalins are released into spinal CSF after repeated noxious stimulation; these could target and activate spinal cord MORs (Le Bars et al., 1987; Bourgoin et al., 1988, 1990; Cesselin et al., 1989). Furthermore, the newly discovered $\mu$-opioid peptides, endomorphin-1 and endomorphin-2, are localized in the spinal cord dorsal horn, in the terminals of small diameter neuropeptide (substance $\mathrm{P}$ and calcitonin gene-related peptide) containing primary afferents (Martin-Schild et al., 1998; Pierce et al., 1998). Because many of the latter are nociceptors, they could provide a source of pain-induced endogenous opioid activity at postsynaptic MORs. To address this question in the present study, we examined the relationship between MOR internalization in lamina II interneurons and MOR-related signaling and behavior. Using MOR internalization as a marker of opioid-induced activity, we also addressed the question as to whether postsynaptic MOR signaling modulates spinal nociceptive processing under normal and inflammatory conditions.

\section{MATERIALS AND METHODS}

Internalization of lamina II MORs in vivo

To establish that MOR internalization occurs and can be observed in lamina II interneurons, we first examined the ability of various exogenously 
applied $\mu$-opioid receptor agonists to internalize the MOR in the spinal cord. The following compounds and injection protocols were used.

Intrathecal injection. Rats were anesthetized with halothane; then DAMGO, morphine, or endomorphin-1 was injected in $20 \mu \mathrm{l}$ of saline with a Hamilton syringe. A 27.5 gauge needle was inserted between the L4/L5 vertebrae above the cauda equina. Intrathecal placement was verified by a flick of the tail on needle entry.

Systemic injection. Morphine was injected subcutaneously at the nape of the neck. In some rats we injected remifentanil, a short-acting potent alkaloid $\mu$-opioid agonist, into one hindpaw.

Morphine pellet implantation. We implanted $75 \mathrm{mg}$ morphine pellets or equivalently sized vehicle pellets subcutaneously at the rear of the flank under halothane anesthesia. We implanted one pellet on day 1, two additional pellets on day 2 , and another three pellets on day 3 . These rats were perfused on day 4 .

\section{Perfusion and tissue preparation}

After treatment the rats were anesthetized deeply with sodium pentobarbital $(100 \mathrm{mg} / \mathrm{kg})$ and then perf used intracardially with $50 \mathrm{ml}$ of $0.1 \mathrm{M}$ PBS, followed by $500 \mathrm{ml}$ of $10 \%$ formalin. The brain and spinal cord were dissected out, post-fixed for $4 \mathrm{hr}$ in the same fixative, and then transferred to a $30 \%$ sucrose solution. Sagittal sections $(30 \mu \mathrm{m})$ of lumbar cord were cut on a freezing microtome.

\section{Immunofluorescent labeling}

Sections were blocked in 5\% normal goat serum in $0.1 \mathrm{M}$ PBS with $0.3 \%$ Triton X-100 for $30 \mathrm{~min}$ and then incubated in a rabbit anti-MOR antibody (Incstar, Stillwater, MN) at a 1:5000 dilution overnight. Sections were washed and incubated in a Cy-3-conjugated goat anti-rabbit antibody (Jackson ImmunoResearch, West Grove, PA) at a 1:600 dilution for $2 \mathrm{hr}$. Then the tissue was washed and mounted on gelatin-coated slides.

\section{Time course of MOR recycling}

To be certain that the stimulus-induced MOR internalization that occurred was indeed detectable, we first established the temporal parameters of internalization, namely, the length of time after ligand binding that MOR internalization could be observed. In these studies we injected 1.0 $\mu \mathrm{g}$ of DAMGO intrathecally and then assayed for analgesia $10 \mathrm{~min}$ after the injection by using the hot plate test. Only rats showing maximal analgesia (60 sec latency) were used for the study. This ensured that $\mu$-opioid receptors were activated by the injection. Immediately after the testing the rats were injected with $1.0 \mathrm{mg} / \mathrm{kg}$ naloxone subcutaneously to prevent further activation of the $\mu$-opioid receptor and thus mark the latest time at which $\mu$-opioid receptor internalization could be induced. To confirm that naloxone had reversed the increase in latency, we again assayed analgesia on the hot plate $5 \mathrm{~min}$ after the naloxone injection. Then the rats were anesthetized with pentobarbital and perfused intracardially with $10 \%$ formalin at $7.5,15,30,60$, or $120 \mathrm{~min}$ after the naloxone injection. Tissue was processed for MOR immunoreactivity as above.

\section{Hot plate test}

Rats were placed in a Plexiglas container in which the floor was heated to $52.5^{\circ} \mathrm{C}$. Behavior was monitored, and the latency until hindpaw licking was determined. Rats were removed from the hot plate as soon as a hindpaw was licked. The cutoff value was $60 \mathrm{sec}$.

\section{Quantification of MOR internalization}

For the in vivo studies we collected confocal images [Nikon $60 \times$ Plan apo (1.40 oil) objective, $2 \times$ zoom, 3.0 iris setting on a Bio-Rad MRC 1024 (Hercules, CA)] through the optical center of 20 MOR-LI lamina II neurons in the L4/L5 segments of each rat. The number of endosomes in each cell was counted from the images by an investigator who was unaware of the treatment that the rat had received. The average number of endosomes per neuron was calculated for each rat. Values are presented as mean \pm SEM.

\section{Correlation between MOR internalization and MOR-induced hyperpolarization}

Although MOR internalization requires agonist binding, the relationship between MOR internalization and MOR-induced activity has not been demonstrated clearly. Because it is difficult to measure directly the opioidinduced internalization and hyperpolarization simultaneously, we mimicked the conditions that have been used to study opioid-induced hyperpolarization in interneurons of lamina II recorded in slice preparations of the dorsal horn (specifically the medullary dorsal horn; Grudt and Williams, 1994). We determined dose-response curves for MOR internalization by using both DAMGO and met-enkephalin, which were the agonists tested in the Grudt and Williams study. $\mathrm{EC}_{50}$ values were calculated with PRISM software (GraphPad Software, San Diego, CA). The curve was a sigmoidal dose-response.

Postnatal day (P) P15-P23 Sprague Dawley rats were anesthetized with halothane, and the lumbar and sacral spinal cord were dissected out. The spinal cord was placed in a bath of carbogenated sucrose buffer (in mM: 240 sucrose, $2.5 \mathrm{KCl}, 1.3 \mathrm{MgSO}_{4}, 1.0 \mathrm{NaH}_{2} \mathrm{PO}_{4}, 10$ glucose, $26 \mathrm{NaHCO}_{3}$, and
2.5 $\mathrm{CaCl}_{2}$ ), and the dura, dorsal, and ventral roots were removed under a dissecting microscope. The cut surface of the lumbar cord was superglued to the stage of a Vibratome (Pelco 101 series 1000), placed against a 5\% agar block, and immersed in carbogenated sucrose buffer. Transverse sections of spinal cord were cut at $500-600 \mu \mathrm{m}$ and transferred to carbogenated incubation solution (in mM: $124 \mathrm{NaCl}, 5 \mathrm{KCl}, 1.3 \mathrm{MgSO}_{4}, 1.2$ $\mathrm{KH}_{2} \mathrm{PO}_{4}, 10$ glucose, $26 \mathrm{NaHCO}_{3}$, and $2.4 \mathrm{CaCl}_{2}$ ) for $2 \mathrm{hr}$. After $2 \mathrm{hr}$ the slices were transferred to a carbogenated recording solution at $37^{\circ} \mathrm{C}$ (in mm: $127 \mathrm{NaCl}, 1.9 \mathrm{KCl}, 1.3 \mathrm{MgSO}_{4}, 1.2 \mathrm{KH}_{2} \mathrm{PO}_{4}, 10$ glucose, $26 \mathrm{NaHCO}_{3}$, and $2.4 \mathrm{CaCl}_{2}$ ) containing the designated concentration of opioid for 15 $\mathrm{min}$. Then the slices were fixed overnight in $10 \%$ formalin and transferred to a $30 \%$ sucrose solution for several hours. Next they were cut at $40 \mu \mathrm{m}$ on a freezing microtome. Immunohistochemistry and quantification of internalization were performed as for the in vivo studies except that confocal images were taken and quantified for only six MOR-LI lamina II neurons from each slice.

\section{Correlation between MOR internalization and behavioral analgesia}

We wished to test the hypothesis that the activation and internalization of MORs on lamina II interneurons are necessary for the analgesia produced by intrathecal $\mu$-opioids. If this hypothesis is correct, then the extent of MOR internalization in lamina II at the lumbar segments innervated by the stimulated dermatome (in this case L4/L5 for the plantar surface of the hindpaw) should correlate with the magnitude of analgesia seen at the dermatome itself. To test this hypothesis, we examined this relationship in individual rats injected intrathecally with various doses of the $\mu$-opioid agonist DAMGO.

Rats were tested on the hot plate test as described above. DAMGO was injected intrathecally in $20 \mu \mathrm{l}$ of saline at doses of $0,10,100$, or $1000 \mathrm{ng}$. Then $15 \mathrm{~min}$ after the injection the rats were tested again on the hot plate test to determine the extent of analgesia produced by the injection. At 25 min after injection the rats were anesthetized with pentobarbital and perfused for immunocytochemistry to visualize and quantify MOR internalization as described above.

Correlation coefficients and $95 \%$ confidence intervals (C.I.) were computed with Statview 4.02 (Abacus Concepts, Berkeley, CA). A Fisher's $r$ to $z$ transformation was used to test the statistical significance of the correlation.

\section{Noxious stimuli}

To determine whether noxious stimuli evoke the release of endogenous $\mu$-opioid agonists that act on lamina II interneurons, we examined MOR internalization in lamina II interneurons throughout the lumbar and cervical spinal cord after exposure of the rat to various noxious stimuli. Stimuli were chosen to mimic those used in studies that detected enkephalin release in spinal CSF (Le Bars et al., 1987; Bourgoin et al., 1988, 1990; Cesselin et al., 1989) or that demonstrated the release of tachykinins from dense core vesicles of primary afferent C-fibers (Abbadie et al., 1997; Liu et al., 1997; Honoré et al., 1999; Trafton et al., 1999). Because endomorphins have been observed in small diameter primary afferents, we assume that these tachykinin-releasing stimuli would release these endogenous opioids also.

Rats were anesthetized with pentobarbital $(50 \mathrm{mg} / \mathrm{kg})$ and exposed to one of the following stimuli to the left hindpaw: 2 min pinch with a hemostat, 2 min immersion in a $52^{\circ} \mathrm{C}$ water bath, alternate immersion and removal from a $52^{\circ} \mathrm{C}$ water bath every $10 \mathrm{sec}$ for 10 or 20 min, interplantar injection of capsaicin $(100 \mu \mathrm{g}$ in $20 \mu \mathrm{l})$, and interplantar injection of $50 \mu \mathrm{l}$ of $5 \%$ formalin. Pinch and water bath stimuli also were assessed $2 \mathrm{~d}$ after injection of $100 \mu \mathrm{l}$ of Freund's complete adjuvant (CFA) into the hindpaw, which produces a persistent inflammation. Rats were perfused 5-15 min after the end of the stimulation. We also evaluated the effect of intrathecal injection of NMDA $(6.8 \mathrm{ng}$ in $20 \mu \mathrm{l})$ and direct electrical stimulation of the sciatic nerve $(10 \mathrm{~Hz}, 0.5 \mathrm{msec} ; 10 \mathrm{~mA}$ for $1 \mathrm{~min})$. Additional time points were tested for the pinch stimulus, with normal rats being perfused at 30 min and CFA-treated rats being perfused at $30 \mathrm{~min}$ and at 1, 2, and $3 \mathrm{hr}$ after stimulus.

\section{RESULTS}

\section{Exogenous opioid-evoked MOR internalization}

To establish that agonist-induced internalization of the MOR occurs in the spinal cord in vivo, we tested a variety of opioid receptor agonists in rats and localized the MOR in lamina II neurons in the spinal cord dorsal horn. We performed immunofluorescent labeling with an antibody against the $\mathrm{C}$-terminal tail of the MOR and examined sagittal sections of lumbar spinal cord. We found that in untreated, saline-injected, or vehicle-pelleted rats MOR-LI was confined to the plasma membrane, with fewer than five MOR-LI-containing endosomes per lamina II neuron (Fig. $1 A)$. In rats that were injected intrathecally with the opioid peptides DAMGO $(1.0 \mu \mathrm{g}$ in $20 \mu \mathrm{l})$ or endomorphin-1 (25 $\mu \mathrm{g}$ in $20 \mu \mathrm{l})$ 25 min before perfusion, MOR-LI was depleted from the plasma 

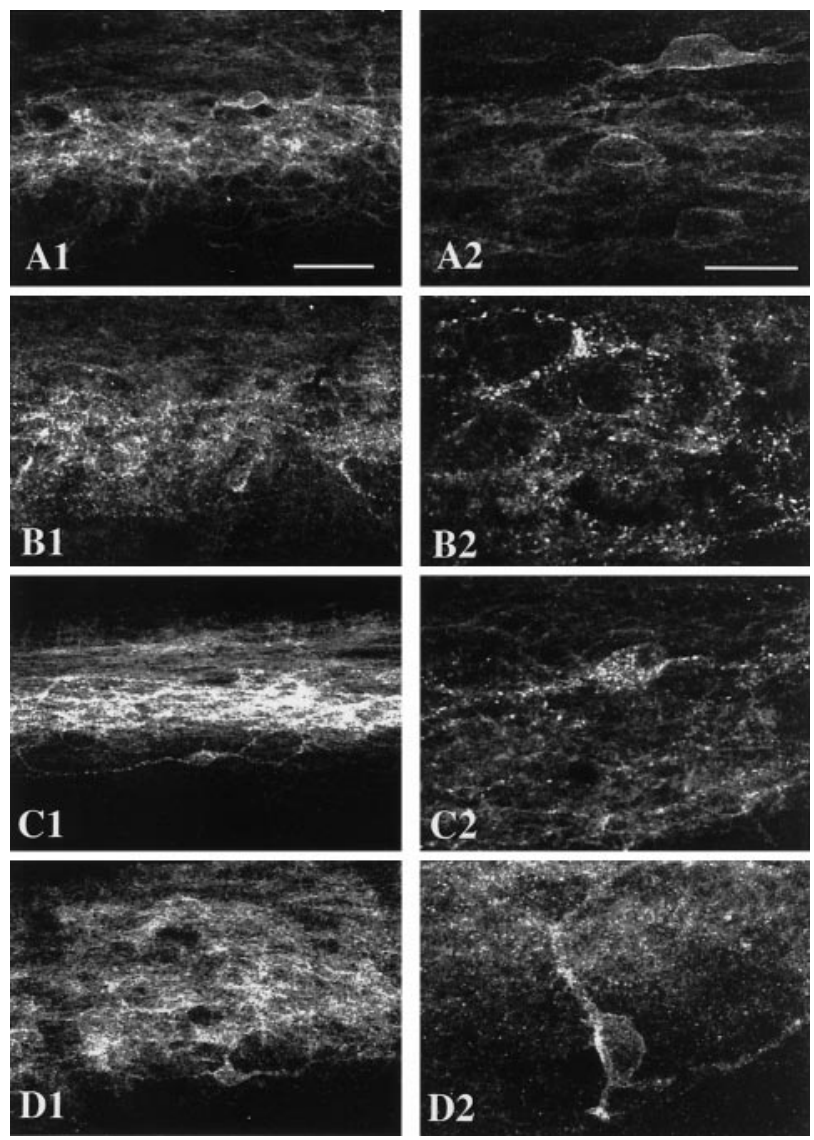

Figure 1. Exogenous opioids, but not morphine, internalize the MOR in lamina II interneurons. Shown are confocal images of lamina II MOR immunoreactivity in the L4/L5 segments of the spinal cord in animals treated with the following: $A$, placebo pellets; $B$, intrathecal endomorphin-1; $C$, intraplantar remifentanil; $D$, morphine pellets (picture 1 is at $35 \times$ and picture 2 is at $105 \times$ magnification). In $A$ and $D$, MOR immunoreactivity is seen predominantly on the cell membranes of neurons and their dendrites, although some smaller endosomes are visible in $D$. In $B$ and $C$, numerous MOR immunoreactive endosomes can be seen in the cell bodies and dendrites of neurons.

membrane and was observed in numerous endosomes within the neuron (Fig. $1 B$ ). Intraplantar (i.e., systemic) injection of remifentanil $(10 \mu \mathrm{g}$ in $50 \mu \mathrm{l})$ produced a comparable pattern of internalization of MOR-LI (Fig. 1C). On the other hand, when we administered morphine systemically $(10 \mathrm{mg} / \mathrm{kg}$, s.c. $)$, intrathecally $(30 \mu \mathrm{g}$ in $20 \mu \mathrm{l}$ ), or subcutaneously via pellets, internalization of the MOR-LI was very limited and generally indistinguishable from control levels (Fig. 1D).

\section{Time course of MOR recycling}

To ensure that MORs did not recycle before we could detect their internalization in response to a stimulus, we believed it was important to establish the time course of the internalization process, specifically how long the receptor remains internalized. To this end we determined the extent of MOR-LI internalization at various times after agonist application; this provided a rough time course of the receptor recycling process. Rats were injected intrathecally with DAMGO; 10 min were allowed for the drug to spread and activate MORs. At this time the rats were given a systemic injection of naloxone to displace DAMGO from the receptor and to prevent further action of any DAMGO that persisted in the CSF. Rats were perfused at various times after the naloxone injection, and the average number of endosomes per lamina II neuron was determined for 20 neurons of the L4 segment. We found that internalization of the MOR peaked at 15 min (Fig. 2). By 60 min the MOR immunoreactivity was indistinguishable from that seen in untreated animals, suggesting that MOR had been recycled to the
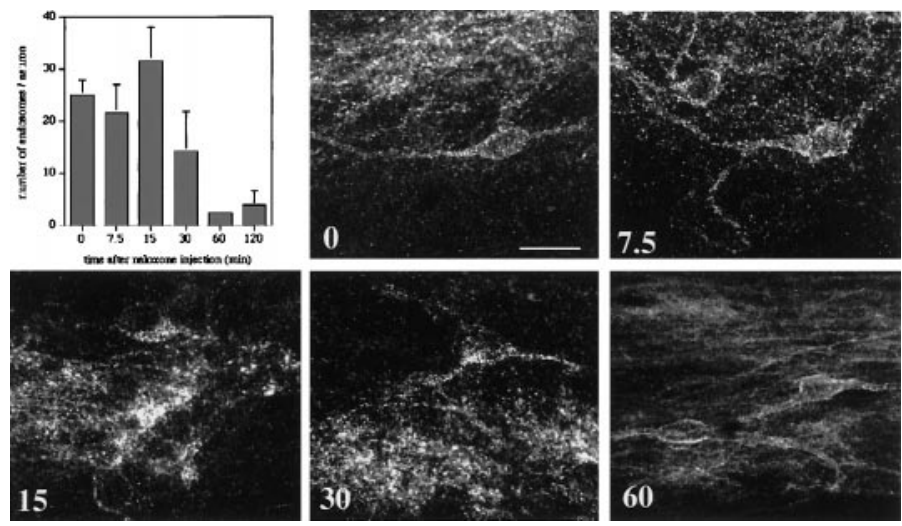

Figure 2. Time course of MOR recycling. The bar graph shows the average number of MOR immunoreactive endosomes found in L4/L5 lamina II neurons in rats that were injected first with $1.0 \mu \mathrm{g}$ of intrathecal DAMGO and $10 \mathrm{~min}$ later with $1.0 \mathrm{mg} / \mathrm{kg}$ naloxone subcutaneously. Quantification was performed at various times after the injection of naloxone $(n=4)$. Also shown are confocal images of lamina II MOR immunoreactivity in the L4/L5 segments of the spinal cord at the indicated times after naloxone injection $(0,7.5,15,30$, and $60 \mathrm{~min})$. MOR internalization can be detected for up to $30 \mathrm{~min}$ after agonist application; note the numerous punctate inclusions in the neurons. MOR immunoreactivity has returned to the plasma membrane by $60 \mathrm{~min}$. Scale bar, $20 \mu \mathrm{m}$.

plasma membrane. Because some MOR internalization was still observed at $30 \mathrm{~min}$, it appears that there is an approximate halfhour window after activation during which MOR internalization can be detected immunocytochemically.

\section{Correlation between MOR internalization and MOR-induced hyperpolarization}

Although the phenomenon of MOR internalization is well documented, the relationship between MOR internalization and opioid function is less clear. $\mu$-Opioids hyperpolarize a subpopulation of lamina II interneurons in the dorsal horn in a dose-dependent manner. Thus, Grudt and Williams (1994) demonstrated that the $\mu$-opioid agonists DAMGO and met-enkephalin induce potassium currents in substantia gelatinosa neurons; the $\mathrm{EC}_{50}$ for DAMGOinduced effects was $72 \pm 12 \mathrm{~nm}(\mathrm{SEM})$. To determine the relationship between MOR internalization and this previously described postsynaptic inhibition of MOR-containing interneurons, we turned to a spinal slice preparation. We determined the doseresponse relationship for DAMGO-induced MOR internalization in these cells, mimicking the conditions that were used in the Grudt and Williams study. We found that bath application of DAMGO resulted in a dose-dependent internalization of the MOR in these neurons $\left(\mathrm{EC}_{50}, 90.96 \mathrm{~nm}\right.$; 95\% C.I., 2.7-3058 nM) (Fig. 3A,C1-C3). This dose-response relationship is similar to that published for inducing potassium currents in lamina II interneurons (Grudt and Williams, 1994). We also examined the dose-response relationship for met-enkephalin-induced MOR internalization. As previously described, fairly high concentrations of met-enkephalin were necessary to activate the MOR (Fig. 3B). Maximal MOR internalization occurred at a concentration of $50 \mu \mathrm{M}$. This is slightly higher than would be predicted on the basis of the single dose $(3.0 \mu \mathrm{M})$ tested in the Grudt and Williams study. However, because peptidase inhibitors were used in the electrophysiological study, somewhat lower doses may have been effective.

\section{Correlation between MOR internalization and opioid- induced behavioral analgesia}

Although the dose-response curve for MOR internalization in lamina II interneurons is comparable with that producing hyperpolarization of a similar population of neurons, the relationship between MOR internalization and the behavioral effects of opioids has not been demonstrated. Thus, to determine whether the internalization of the MOR in lamina II of the spinal cord correlates with spinal opioid-induced analgesia, we compared the magnitude 

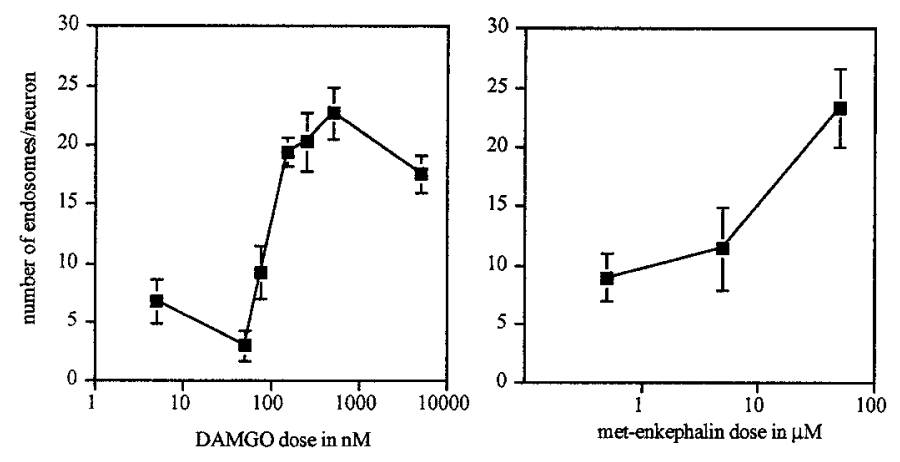

Figure 3. Opioid receptor internalization occurs in a dose-dependent manner. Slices of spinal cord were incubated in various doses of DAMGO (left) or met-enkephalin (right) for $15 \mathrm{~min}$, and the average number of MOR immunoreactive endosomes per lamina II neuron was determined. Untreated control slices had $6.53 \pm 1.16$ endosomes per neuron for the DAMGO experiments and $9.17 \pm 0.76$ endosomes per neuron for the enkephalin experiments ( $n=4-9$ for DAMGO; $n=5$ for met-enkephalin).

of MOR internalization in lamina II neurons with the magnitude of behavioral analgesia on the hot plate test after intrathecal injection of DAMGO. We found that the dose-response relationship for internalization of the MOR in lamina II of the lumbar spinal cord was remarkably similar to that which plotted analgesia in the hot plate test (Fig. 4A). Furthermore, there was a high correlation between the magnitude of analgesia and the average number of endosomes per lamina II neuron of the lumbar spinal cord seen in individual rats [Fig. 4B; correlation coefficient, 0.913 (95\% C.I., $0.807-0.962) ; p=<0.0001]$.

\section{Noxious stimuli}

Several studies have demonstrated that endogenous opioids are released in the spinal cord after noxious stimuli (Yaksh and Elde, 1981; Le Bars et al., 1987; Bourgoin et al., 1988, 1990). Here we examined whether noxious stimulation also induces MOR internalization in lamina II interneurons. In these studies we monitored MOR-LI in lamina II of lumbar spinal cord after noxious stimuli of various modalities. We chose stimulus paradigms that modeled those used in studies that demonstrated spinal cord release of enkephalins or primary afferent neuropeptides. In none of these experiments did we detect significant MOR internalization (Fig. 5).

It is significant that each of these stimuli induced internalization of the NK-1 receptor in lamina I of the spinal cord in the same animals (data not shown; but see Liu et al., 1997; Abbadie et al., 1997; Honoré et al., 1999; Trafton et al., 1999). We are, therefore, confident that the stimuli were of sufficient intensity to release dense core vesicles from at least a subpopulation of small diameter primary afferents. Because nonsegmental enkephalin release has been reported (Le Bars et al., 1987), we also examined sagittal sections of both lumbar and cervical spinal cord. Regardless of the areas that were examined, we could not distinguish MOR localization in lamina II spinal cord neurons from animals that were treated as described (see Materials and Methods) from those in untreated animals.

Because there is evidence for enhanced release of opioids during peripheral inflammation, we also studied MOR internalization under these conditions. Again, none of the stimuli that were tested induced any detectable MOR internalization in the spinal cord (Fig. 6). Among the stimuli that were used was intraplantar injection of $5 \%$ formalin, a stimulus that produces both inflammation and pain behavior. Noxious stimuli in the presence of fully developed inflammation were also ineffective. Furthermore, MOR internalization was not observed after the induction of profound inflammation of the hindpaw and ankle with CFA. Even superimposed on these inflammatory conditions, a noxious pinch of the hindpaw for $2 \mathrm{~min}$ (at 5, 30, 120, or $180 \mathrm{~min}$ ) or immersion of the hindpaw in a $52^{\circ} \mathrm{C}$ water bath for $2 \mathrm{~min}$ (at $5 \mathrm{~min}$ ) did not induce MOR internalization.

\section{DISCUSSION}

In the present study we show that MOR internalization occurs in the spinal cord in a manner comparable with that observed in the hypothalamus (Eckersell et al., 1998), in the enteric nervous system (Sternini et al., 1996) and in cultured cells (Arden et al., 1995; Keith et al., 1996). We could detect significant MOR internalization after the administration of opioids locally or systemically. However, as previously described (Keith et al., 1996), morphine did not induce comparable internalization of the MOR, even with long exposures or when high doses were used. Importantly, all endogenous opioids as well as most nonmorphine alkaloid agonists (such as remifentanil) tested in this and other studies (Burford et al., 1998; Keith et al., 1998; McConalogue et al., 1999) induced the internalization of MORs. Thus, although it is possible for an opioid to induce MOR signaling without internalization (specifically morphine), it is unlikely that endogenous MOR ligands would signal via the MOR without triggering visible internalization and recycling.

We found that the MOR is internalized rapidly and recycles to the plasma membrane within $\sim 60 \mathrm{~min}$. This time course is similar to that observed for other G-protein-coupled receptors (Mantyh et al., 1995) and suggests that there is an $\sim 30$ min time window in which receptor activation can be detected. Importantly, this relatively prolonged window of recycling time ensures that we did not miss any significant MOR internalization that might have occurred after the release of endogenous opioids. The animals were perfused long before the receptor would have recycled completely.

Of additional note, we found that the behavioral analgesia produced by intrathecal DAMGO injection is maintained for at least $30 \mathrm{~min}$, yet it can be reversed fully by naloxone within minutes. Recycling of the MOR, however, is not completed for $>30 \mathrm{~min}$ after naloxone treatment. The fact that naloxone can reverse the analgesia produced by DAMGO much more quickly than the receptors appear to recycle suggests that the prolongation of DAMGO analgesia requires ongoing interactions with and activation of the MOR. This result also indicates that the duration of the DAMGO analgesia is not the result of extended second messenger signaling or activation of a circuit triggered by an initial interaction with MORs. It follows from this conclusion that not all MORs on a given neuron are desensitized functionally during the period in which most of the immunoreactivity appears to be endosomal. Thus, despite the internalization of a large population of MORs, a significant number of nondesensitized receptors likely remain at the plasma membrane, even in the presence of high doses of a high efficacy agonist.

\section{Relationship between MOR internalization and MOR signaling}

The similarity between the dose-response curves obtained here for DAMGO- and enkephalin-induced MOR internalization and those previously described for MOR-activated potassium currents suggests that MOR internalization provides a useful and accurate functional marker of MOR-induced signaling. In fact, analysis of the dose-response curves obtained for MOR-induced hyperpolarization (Grudt and Williams, 1994) suggests that MOR internalization is as good a marker of MOR signaling as intracellular recordings and can indicate whether postsynaptic inhibition is occurring by the mechanisms that have been proposed. It is, of course, possible that some consequences of MOR activation occur that we cannot detect by monitoring internalization; however, this is probably below the threshold for inducing potassium conductance and thus should not affect the membrane potential of the neuron significantly. Using a slightly different quantification method (counting the percentage of neurons with MOR internalization rather than number of MOR-positive endosomes), Marvizon et al. (1999) recently reported a similar dose-response for DAMGO-induced MOR internalization in the spinal cord slice $\left(\mathrm{EC}_{50}, 30 \mathrm{nM}\right)$. As these authors pointed out, the $\mathrm{EC}_{50}$ values for MOR internalization in the spinal cord slice are nearly identical to those obtained for adenyl cyclase inhibition or $\left[\gamma^{-35}\right.$ S GTP binding in cell culture systems (Keith et al., 1996, 1998; Yabaluri and 
Figure 4. Lamina II MOR internalization correlates with intrathecal DAMGO-induced analgesia in the hot plate test. Rats were injected with various doses of intrathecal DAMGO, tested for analgesia on the hot plate test, and then anesthetized and perfused for immunocytochemistry. MOR internalization was quantified in lamina II neurons from the L4/L5 segment of the spinal cord. A, Dose-response curves for changes in hot plate latency and the extent of MOR internalization. DAMGO produces spinal MOR internalization and behavioral analgesia at the same doses. Saline-injected rats had $5.58 \pm 1.17$ endosomes per neuron $(n=6)$. $B$, Graph of the magnitude of analgesia (hot plate latency in seconds) versus the extent of MOR internalization (number of endosomes per neuron) in lamina II of the L4/L5 segment in individual rats. Only rats that were analgesic showed significant MOR internalization. $C$, Confocal images of MOR immunoreactivity in lamina II from rats injected intrathecally with saline (1), $100 \mathrm{ng}$ of DAMGO (2), or 1000 ng of DAMGO (3). MOR immunoreactivity is observed on the plasma membrane in vehicletreated rats. Increasing doses of DAMGO produced increases in the amount of MOR immunoreactivity that appeared as endosomal structures in the cytoplasm.
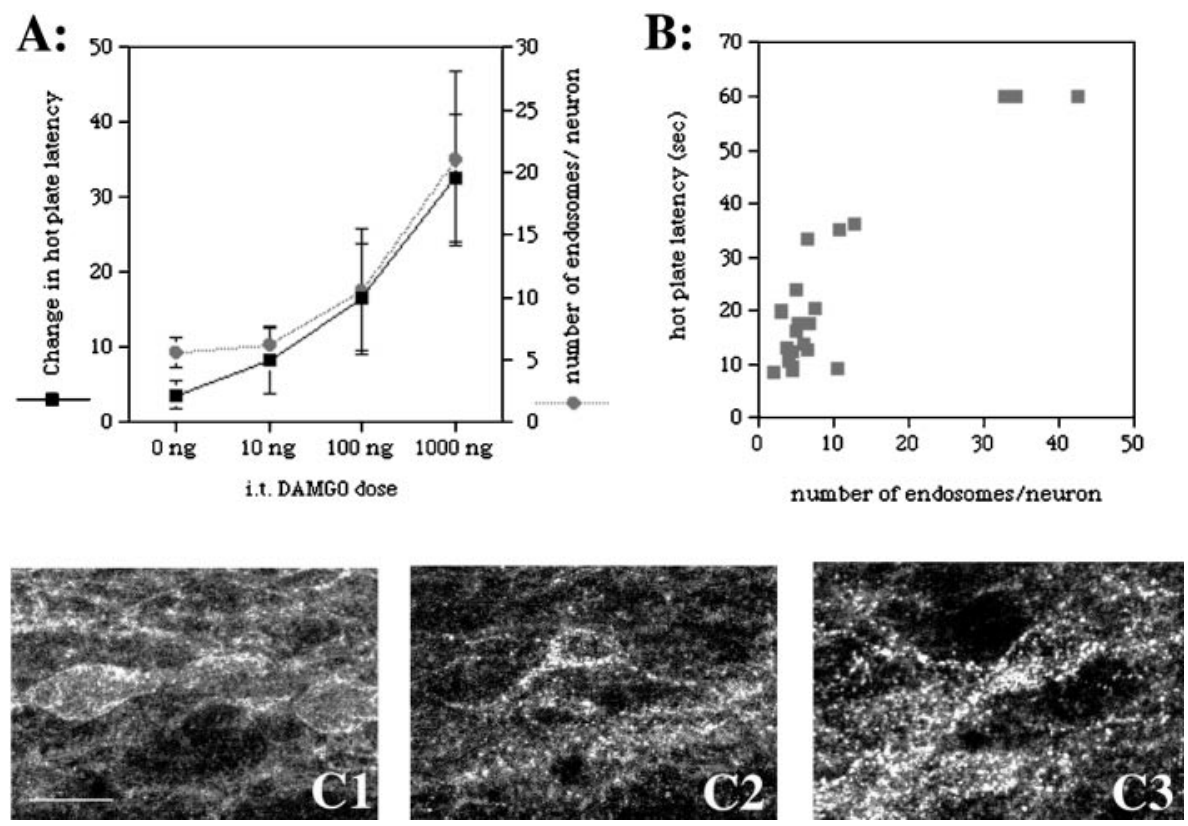
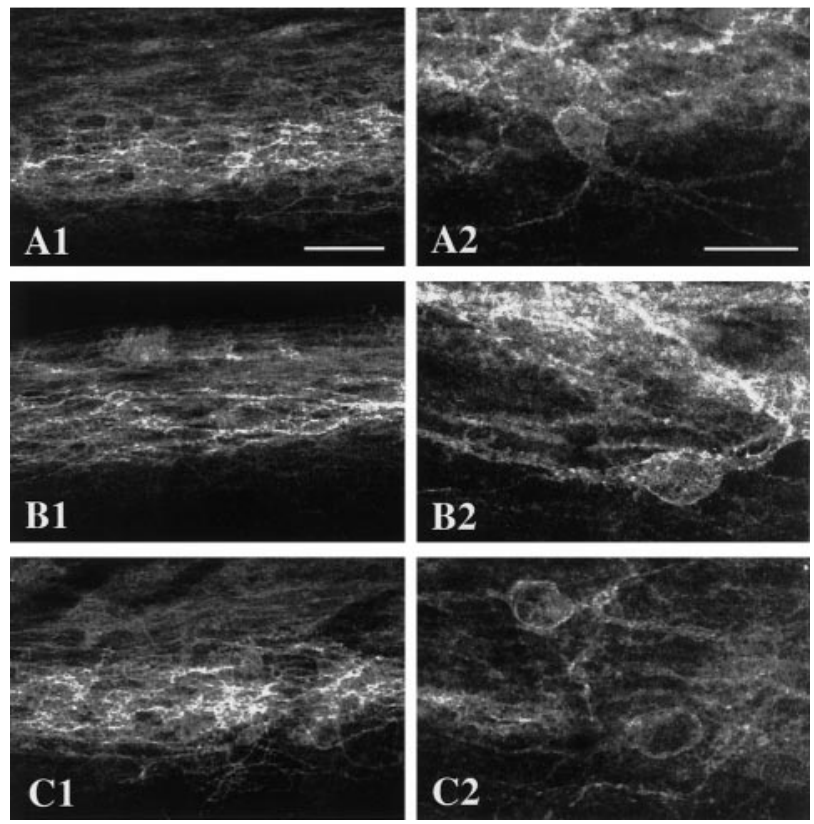

Figure 5. Noxious stimuli do not internalize the MOR. Noxious stimul were applied to a hindlimb of an anesthetized rat. The rats were perfused 5-30 min later. Shown are confocal images of MOR immunoreactivity in lamina II of the L4/L5 segments of the spinal cord ipsilateral to the stimulus. $A$, This rat received a noxious pinch of the hindpaw. $B$, This rat received an intraplantar injection of capsaicin. $C$, The hindpaw of this rat was alternatively dipped and removed from $52^{\circ} \mathrm{C}$ water every $10 \mathrm{sec}$ for 10 min (Picture 1 is at $35 \times$, scale bar, $50 \mu \mathrm{m}$; picture 2 is at $105 \times$, scale bar, $20 \mu \mathrm{m})$.

Medzihradsky, 1997). These data provide further evidence that MOR internalization can be used as a functional marker of MOR activation.

DAMGO-induced MOR internalization correlated exceptionally well with DAMGO-induced hot plate analgesia. Indeed, only when rats were rendered analgesic by intrathecal DAMGO did we observe MOR internalization. This result suggests that postsynaptic MOR-mediated hyperpolarization of excitatory interneurons does occur and likely is involved in the behavioral analgesia produced by exogenous opioids. Of course, primary afferent-derived MORs are nearby and thus probably also would be exposed to DAMGO that accessed this region of the cord. In fact, if the
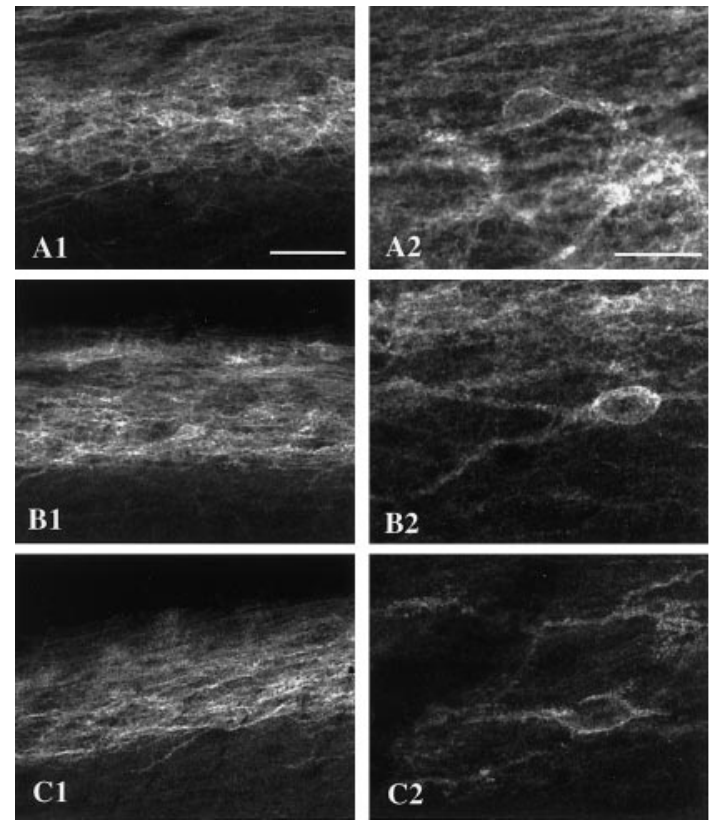

Figure 6. Noxious stimuli do not internalize the MOR under conditions of inflammation. Shown are confocal images of MOR immunoreactivity in lamina II of the L4/L5 segments of the spinal cord ipsilateral to the inflammation and/or stimulus. $A$, This rat received an intraplantar injection of $5 \%$ formalin. $B$, This rat received a noxious pinch to a hindpaw that was inflamed after the injection of CFA, $2 \mathrm{~d}$ earlier. $C$, The hindpaw of this rat was dipped in a $52^{\circ} \mathrm{C}$ water bath for $2 \mathrm{~min}, 2 \mathrm{~d}$ after an injection of CFA (Picture 1 is at $35 \times$, scale bar, $50 \mu \mathrm{m}$; picture 2 is at $105 \times$, scale bar, $20 \mu \mathrm{m})$.

primary afferent opioid receptor terminals were critical for the induction of spinal analgesia, then MOR internalization in lamina II neurons might correlate with, but not be critical to, the behavioral analgesia. The presence of internalization would indicate merely that sufficient drug had reached the appropriate region of the spinal cord. Nevertheless, our data, as well as our previous study of opioid effects on noxious stimulus-evoked tachykinin signaling (Trafton et al., 1999), are consistent with the hypothesis that lamina II postsynaptic MORs are key mediators of the analgesia that is produced by spinal opioids.

The close correlations between G-protein-coupled receptor internalization and functional activity that are found here are not 
unprecedented. Keith et al. (1998) found similar dose-response relationships for DAMGO- and etorphine-induced MOR internalization and the inhibition of forskolin-stimulated cAMP accumulation in stably transfected HEK-293 cells. We also found a tight correlation between tachykinin-induced NK-1 receptor internalization and tachykinin-induced increases in intracellular calcium in cultured spinal cord neurons (Trafton et al., 1999). Additionally, it has been suggested that MOR internalization is linked to some aspects of its signaling, specifically the activation of MAP kinase (Polakiewicz et al., 1998; Ignatova et al., 1999), demonstrating that internalization and activity may be related functionally, not just correlated temporally.

\section{Consequences of noxious stimulation}

Several studies suggested that noxious stimulation evokes the release of endogenous opioids into the spinal CSF. Nevertheless, we found no indication that MORs on interneurons in lamina II are internalized/activated by noxious stimulation. If endomorphins are released from primary afferent fibers during noxious stimuli, it seems that their activity is concentrated and possibly restricted to MORs on primary afferent terminals. Because MORs have been observed on the terminals of small diameter fibers that contain endomorphins (Martin-Schild et al., 1998), it follows that these endomorphins could act at a primary afferent autoreceptor, which would restrict further the $\mathrm{C}$-fiber input. Based on our inability to detect opioid receptor internalization, these endomorphins do not appear to have postsynaptic effects in lamina II, unless their target postsynaptic neurons express a MOR splice variant that is not recognized by the antisera that have been used in the present study (Pan et al., 1999).

Our results also suggest that the met-enkephalin release that has been detected after noxious stimulation (Le Bars et al., 1987; Bourgoin et al., 1988, 1990; Cesselin et al., 1989) does not evoke postsynaptic effects via the MOR. Because the $\delta$-opioid receptor (DOR) is found in the superficial dorsal horn (Arvidsson et al., 1995b) and because enkephalins have a higher affinity for DOR (Corbett et al., 1993), one possibility is that locally released enkephalins primarily activate this opioid receptor subtype. In support of this hypothesis, Mizoguchi et al. (1997) have shown that intrathecal met-enkephalin-induced analgesia is blocked by the $\delta_{2}$-opioid receptor antagonist naltriben, but not by the MOR antagonist CTOP. Alternatively, it is possible that noxious stimulation does not release opioid peptides in sufficient quantity to activate any opioid receptors. This possibility is consistent with the lack of effect of the general opioid receptor antagonist naloxone on nociceptive behaviors in animals or pain sensation in humans, in a number of acute pain models (El-Sobky et al., 1976; Goldstein et al., 1976; Grevert and Goldstein, 1978; North, 1978; Stacher et al., 1988). Also, mice lacking the MOR have been shown to have normal behavioral responses to acute noxious thermal stimuli (Matthes et al., 1996), further suggesting that endogenous $\mu$-opioids are not involved in normal acute nociceptive responses.

Several studies reported that opioids are more effective under inflammatory conditions (Neil et al., 1986; Millan et al., 1988), and there is evidence that levels of enkephalin in the dorsal horn increase with peripheral inflammation (Millan et al., 1986; Iadarola et al., 1988). These results raised the possibility that greater amounts of enkephalin may be released and/or activate opioid receptors under inflammatory conditions (however, see Pohl et al., 1997). In fact, we found no indication of basal or noxious stimulusevoked MOR signaling in the presence of inflammation. Therefore, it appears that, even under these conditions of hypersensitivity, spinal postsynaptic MORs are not activated endogenously.

Given that we found a profound analgesia associated with MOR internalization in lamina II interneurons, we suggest that this significant MOR internalization does not and indeed should not occur during normal or injury-related nociceptive responses. Were MORs activated to such an extent in vivo, responses to noxious stimuli would be blocked effectively, preventing the withdrawal from and attendance to stimuli that potentially are damaging. This is clearly undesirable and potentially life-threatening, particularly if the inhibition were a constant response to noxious stimulation.

The question remains as to the conditions under which the activation of MORs on lamina II interneurons occurs in vivo. Although it clearly is associated with the analgesia produced by exogenous opioids, one presumes that these receptors have a physiological/natural function. Given the evidence for an endogenous opioid contribution to the modulation of nociceptive behaviors, a function in stress- or sex-induced analgesia is a reasonable consideration, particularly because inattention to pain in these conditions might be beneficial to survival or reproduction.

\section{REFERENCES}

Abbadie C, Trafton J, Liu H, Mantyh PW, Basbaum AI (1997) Inflammation increases the distribution of dorsal horn neurons that internalize the neurokinin-1 receptor in response to noxious and non-noxious stimulation. J Neurosci 17:8049-8060.

Arden JR, Segredo V, Wang Z, Lameh J, Sadee W (1995) Phosphorylation and agonist-specific intracellular trafficking of an epitope-tagged $\mu$-opioid receptor expressed in HEK-293 cells. J Neurochem 65:1636-1645.

Arvidsson U, Riedl M, Chakrabarti S, Lee J, Nakano A, Dado R, Loh H, Law P, Wessendorf M. Elde R (1995a) Distribution and targeting of a $\mu$-opioid receptor (MOR1) in brain and spinal cord. J Neurosci 15:3328-3341.

Arvidsson U, Dado RJ, Riedl M, Lee JH, Law PY, Loh HH, Elde R, Wessendorf MW (1995b) $\delta$-Opioid receptor immunoreactivity: distribution in brainstem and spinal cord, and relationship to biogenic amines and enkephalin. J Neurosci 15:1215-1235.

Bourgoin S, Le Bars D, Clot AM, Hamon M, Cesselin F (1988) Spontaneous and evoked release of met-enkephalin-like material from the spinal cord of arthritic rats in vivo. Pain 32:107-114.

Bourgoin S, Le Bars D, Clot AM, Hamon M, Cesselin F (1990) Subcutaneous formalin induces a segmental release of met-enkephalin-like material from the rat spinal cord. Pain 41:323-329.

Burford NT, Tolbert LM, Sadee W (1998) Specific G-protein activation and $\mu$-opioid receptor internalization caused by morphine, DAMGO, and endomorphin I. Eur J Pharmacol 342:123-126.

Cesselin F, Bourgoin S, Clot A, Hamon M, Le Bars D (1989) Segmental release of met-enkephalin-like material from the spinal cord of rats, elicited by noxious thermal stimuli. Brain Res 484:71-77.

Corbett AD, Paterson SJ, Kosterlitz HW (1993) Selectivity of ligands for opioid receptors. In: Opioids I (Herz A, ed), pp 645-679. Berlin: Springer.

Eckersell CB, Popper P, Micevych PE (1998) Estrogen-induced alteration of $\mu$-opioid receptor immunoreactivity in the medial preoptic nucleus and medial amygdala. J Neurosci 18:3967-3976.

El-Sobky A, Dostrovsky JO, Wall PD (1976) Lack of effect of naloxone on pain perception in humans. Nature 263:783-784.

Goldstein A, Pryor GT, Otis LS, Larsen F (1976) On the role of endogenous opioid peptides: failure of naloxone to influence shock escape threshold in the rat. Life Sci 18:599-604.

Grevert P, Goldstein A (1978) Endorphins: naloxone fails to alter experimental pain or mood in humans. Science 199:1093-1095.

Grudt T, Williams JT (1994) $\mu$-Opioid agonists inhibit spinal trigeminal substantia gelatinosa neurons in guinea pig and rat. $J$ Neurosci 14:1646-1654.

Honoré P, Menning P, Rogers S, Nichols M, Basbaum AI, Besson JM, Mantyh PW (1999) Spinal substance P receptor expression and internalization in acute, short-term, and long-term inflammatory pain states. J Neurosci 19:7670-7678.

Iadarola MJ, Douglass J, Civelli O, Naranjo JR (1988) Differential activation of spinal cord dynorphin and enkephalin neurons during hyperalgesia: evidence using cDNA hybridization. Brain Res 455:205-212.

Ignatova EG, Belcheva MM, Bohn LM, Neuman MC, Coscia CJ (1999) Requirement of receptor internalization for opioid stimulation of mitogen-activated protein kinase: biochemical and immunofluorescence confocal microscopic evidence. J Neurosci 19:56-63.

Keith D, Murray S, Zaki P, Chu P, Lissin D, Kang L, Evans C, Von Zastrow M (1996) Morphine activates opioid receptors without causing their rapid internalization. J Biol Chem 271:19021-19024.

Keith D, Anton B, Murray S, Zaki P, Chu P, Lissin D, Monteileet-Agius G, Stewart P, Evans C, Von Zastrow M (1998) $\mu$-Opioid receptor internalization: opiate drugs have differential effects on a conserved endocytic mechanism in vitro and in the mammalian brain. Mol Pharmacol 53:377-384.

Le Bars D, Bourgoin S, Clot AM, Hamon M, Cesselin F (1987) Noxious mechanical stimuli increase the release of met-enkephalin-like material heterosegmentally in the rat spinal cord. Brain Res 402:188-192.

Liu H, Mantyh PW, Basbaum AI (1997) NMDA receptor regulation of substance $\mathrm{P}$ release from primary afferent nociceptors. Nature 386:721-724.

Mantyh PW, DeMaster E, Malhotra A, Ghilardi JR, Rogers SD, Mantyh CR, Liu H, Basbaum AI, Vigna SR, Maggio JE, Simone DA (1995) 
Receptor endocytosis and dendrite reshaping in spinal neurons after somatosensory stimulation. Science 268:1629-1632.

Martin-Schild S, Gerall AA, Kastin AJ, Zadina JE (1998) Endomorphin-2 is an endogenous opioid in primary sensory afferent fibers. Peptides 19:1783-1789.

Marvizon JC, Grady EF, Waszak-McGee J, Mayer EA (1999) Internalization of $\mu$-opioid receptors in rat sinal cord slices. NeuroReport 10:2329-2334.

Matthes HW, Maldonado R, Simonin F, Valverde O, Slowe S, Kitchen I, Befort K, Dierich A, Le Meur M, Dolle P, Tzavara E, Hanoune J, Roques BP, Keiffer BL (1996) Loss of morphine-induced analgesia reward effect, and withdrawal symptoms in mice lacking the $\mu$-opioid receptor gene. Nature 383:819-823.

McConalogue K, Grady EF, Minnis J, Balestra B, Tonini M, Brecha NC, Bunnett NW, Sternini C (1999) Activation and internalization of the $\mu$-opioid receptor by the newly discovered endogenous agonists, endomorphin-1 and endomorphin-2. Neuroscience 90:1051-1059.

Millan MJ, Millan MH, Czlonkowski A, Hollt V, Pilcher CW, Herz A, Colpaert FC (1986) A model of chronic pain in the rat: response of multiple opioid systems to adjuvant-induced arthritis. J Neurosci 6:899-906.

Millan MJ, Czlonkowski A, Morris B, Stein C, Arendt, Huber A, Hollt V, Herz A (1988) Inflammation of the hind limb as a model of unilateral, localized pain: influence on multiple opioid systems in the spinal cord of the rat. Pain 35:299-312.

Mizoguchi H, Narita M, Kampine JP, Tseng LF (1997) [Met ${ }^{5}$ ]enkephalin and $\delta_{2}$-opioid receptors in the spinal cord are involved in the cold water swimming-induced antinociception in the mouse. Life Sci 61:PL81-PL86.

Neil A, Kayser V, Gacel G, Besson JM, Guilbaud G (1986) Opioid receptor types and antinociceptive activity in chronic inflammation: both $\kappa$ and $\mu$-opiate agonistic effects are enhanced in arthritic rats. Eur J Pharmacol 130:203-208.
North MA (1978) Naloxone reversal of morphine analgesia but failure to alter reactivity to pain in the formalin test. Life Sci 22:295-302.

Pan YX, Xu J, Bolan E, Abbadie C, Chang A, Zuckerman A, Rossi G, Pasternak GW (1999) Identification and characterization of three new alternatively spliced mu-opioid receptor isoforms. Mol Pharmacol 56: 396-403.

Pierce TL, Grahek MD, Wessendorf MW (1998) Immunoreactivity for endomorphin-2 occurs in primary afferents in rats and monkey. NeuroReport 9:385-389.

Pohl M, Ballet S, Collin E, Mauborgne A, Bourgoin S, Benoliel JJ, Hamon M, Cesselin F (1997) Enkephalinergic and dynorphinergic neurons in the spinal cord and dorsal root ganglia of the polyarthritic rat: in vivo release and cDNA hybridization studies. Brain Res 749:18-28.

Polakiewicz RD, Schieferl SM, Dorner LF, Kansra V, Comb MJ (1998) A mitogen-activated protein kinase pathway is required for $\mu$-opioid receptor desensitization. J Biol Chem 273:12402-12406.

Stacher G, Abatzi TA, Schulte F, Schneider C, Stacher-Janotta G, Gaupmann G, Mittelbach G, Steinringer H (1988) Naloxone does not alter the perception of pain induced by electrical and thermal stimulation of the skin in healthy humans. Pain 34:271-276.

Sternini C, Spann M, Anton B, Keith D, Bunnett N, Von Zastrow M, Evans C, Brecha N (1996) Agonist-selective endocytosis of $\mu$-opioid receptor by neurons in vivo. Proc Natl Acad Sci USA 93:9241-9246.

Trafton JA, Abbadie C, Marchand S, Mantyh PW, Basbaum AI (1999) Spinal opioid analgesia: how critical is the regulation of substance $\mathrm{P}$ signaling? J Neurosci 19:9642-9653.

Yabaluri N, Medzihradsky F (1997) Down-regulation of $\mu$-opioid receptor by full, but not partial, agonists is independent of G-protein coupling. Mol Pharmacol 52:896-902.

Yaksh TL, Elde RP (1981) Factors governing release of methionine enkephalin-like immunoreactivity from mesencephalon and spinal cord of the cat in vivo. J Neurophysiol 46:1056-1075. 